\title{
Absolute measurement of the resonance lines in heliumlike vanadium on an electron-beam ion trap
}

\author{
C. T. Chantler, ${ }^{1}$ D. Paterson, ${ }^{1}$ L. T. Hudson, ${ }^{2}$ F. G. Serpa, ${ }^{2}$ J. D. Gillaspy, ${ }^{2}$ and E. Takács ${ }^{2, *}$ \\ ${ }^{1}$ School of Physics, University of Melbourne, 3010, Australia \\ ${ }^{2}$ National Institute of Standards and Technology, Gaithersburg, Maryland 20899
}

(Received 14 February 2000; published 11 September 2000)

\begin{abstract}
Absolute measurements of the energies of resonance lines in heliumlike vanadium on an electron-beam ion trap are reported. The $1 s 2 p^{1} P_{1} \rightarrow 1 s^{2}, 1 s 2 p^{3} P_{2} \rightarrow 1 s^{2}\left(1 s 2 p^{3} P_{1} \rightarrow 1 s^{2}\right.$ and $\left.1 s 2 p^{3} P_{0} \rightarrow 1 s^{2}\right)$ blend and $1 s 2 s^{3} S_{1} \rightarrow 1 s^{2}$ transitions are $5205.10 \pm 0.14 \mathrm{eV}, 5189.12 \pm 0.21 \mathrm{eV}, 5180.22 \pm 0.17 \mathrm{eV}$, and 5153.82 $\pm 0.14 \mathrm{eV}$, respectively. This agrees with recent theoretical calculations and the experimental precision lies at the same level as the current uncertainty in theory $(0.1 \mathrm{eV})$. These measurements represent a $5.7-8 \%$ determination of the QED contribution to the transition energies and are the most precise measurements of heliumlike resonance lines in the $Z=19-31$ range. The measurement of the $1 s 2 s^{3} S_{1} \rightarrow 1 s^{2}$ transition is also sensitive to the $1 s 2 s{ }^{3} S_{1}$ QED contribution at the $40 \%$ level. The intensity of the strong $1 s 2 p{ }^{3} P_{1} \rightarrow 1 s^{2}$ component of the blend compared to the total blend intensity is $94 \% \pm 12 \%$. This is in accord with current theoretical predictions but disagrees with an earlier reported ratio.
\end{abstract}

PACS number(s): 31.30.Jv, 32.30.Rj, 12.20.Fv, 34.80.Kw

\section{INTRODUCTION AND BACKGROUND}

The calculation of two-electron ion energy levels has been a topic of much research since the discovery of quantum mechanics. The contribution of relativistic and quantum electrodynamic (QED) effects has been intensely studied in the last three decades $[1,2]$. Two-electron systems provide a test bed for not only quantum electrodynamics and relativistic effects calculations, but also for many-body formalisms [3].

Theoretical calculations of energy levels in heliumlike ions using variational techniques, relativistic corrections, and a $Z \alpha$ expansion in the unified approach were pioneered by Drake in 1979 and 1988 [4,5]. Since then there has been active research in the calculation of energy levels in heliumlike ions using new techniques. Major progress in theoretical calculations of QED contributions has occurred in the last decade, particularly in developments avoiding the $Z \alpha$ expansion by using all-orders techniques [6-9]. Plante et al. [6] provide a recent review of calculations of the $n=1$ and $n$ $=2$ states of heliumlike ions and present relativistic all-order many-body calculations.

To critically test QED contributions at the $10 \%$ or better level in medium $Z$ ions requires an experimental precision of 10-30 ppm. In many cases experiments of sufficient precision have not been performed which could resolve the differences between theories $[6,9,10]$. Repeated claims in the literature have suggested that across the $Z=19-32$ range experimental determinations of the $1 s 2 p^{1} P_{1} \rightarrow 1 s^{2}$ transition energy are greater than theory $[11-13]$. We have directed our research at an absolute precision measurement of heliumlike resonance lines in vanadium $(Z=23)$ to investigate these claims in the center of the region of interest (heliumlike resonance lines refer to $n=2$ to $n=1$ transitions in two-electron ions where one electron remains in the ground $(1 s)$ state as illustrated in Fig. 1). No prior high precision

\footnotetext{
*Permanent address: Institute of Experimental Physics, University of Debrecen, Debrecen, Bemter 18/a, H-4026 Hungary.
}

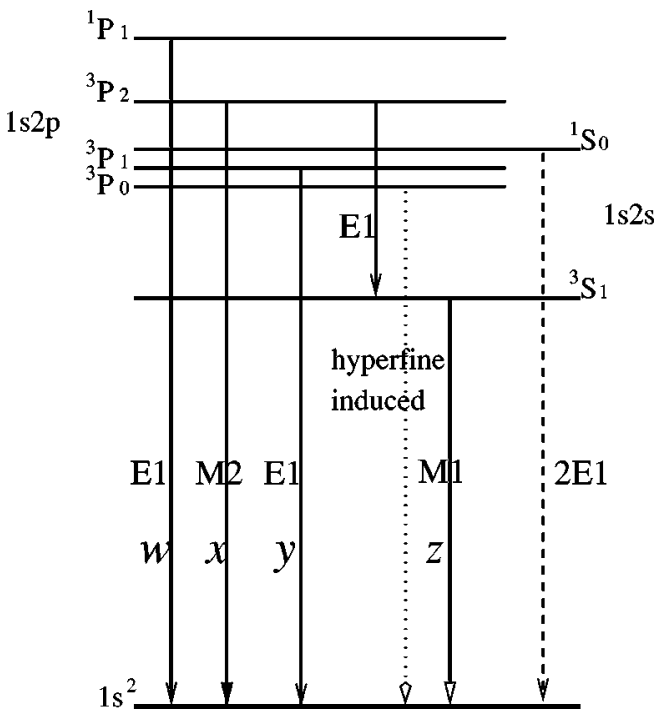

FIG. 1. Radiative decay scheme of medium $Z$ heliumlike ions, indicating the nature of decay: $E 1$ electric dipole, $M 1$ magnetic dipole, $M 2$ magnetic quadrupole radiation, and $2 E 1$ two-photon $E 1$ process. The hyperfine-induced $1 s 2 p^{3} P_{0} \rightarrow 1 s^{2}$ transition is only allowed if the nuclear magnetic moment is finite (vanadium $7 / 2 \hbar$ ). The notation of Gabriel [14] is indicated for each resonance line.

absolute measurements in vanadium have been performed, which was an additional motivation. For the $1 s 2 p{ }^{1} P_{1}$ $\rightarrow 1 s^{2}$ transition ( $w$ line, notation of Gabriel [14]) it has been claimed that the heliumlike resonance line calculated by Drake is too large by about $60 \mathrm{ppm}$ [12] based upon a series of measurements of the $w$ line $(Z=19-26)$ [11]. The QED contributions to the heliumlike resonance lines in vanadium are 480-550 ppm of the transition energies [5] and therefore precise measurements (better than $30 \mathrm{ppm}$ ) of these transitions are sensitive to, and can test, calculations of the QED component.

The uncertainty of theoretical determinations of energy states in two-electron ions has been a focus of recent attention [10]. Earlier theoretical work [5] claimed an uncertainty 


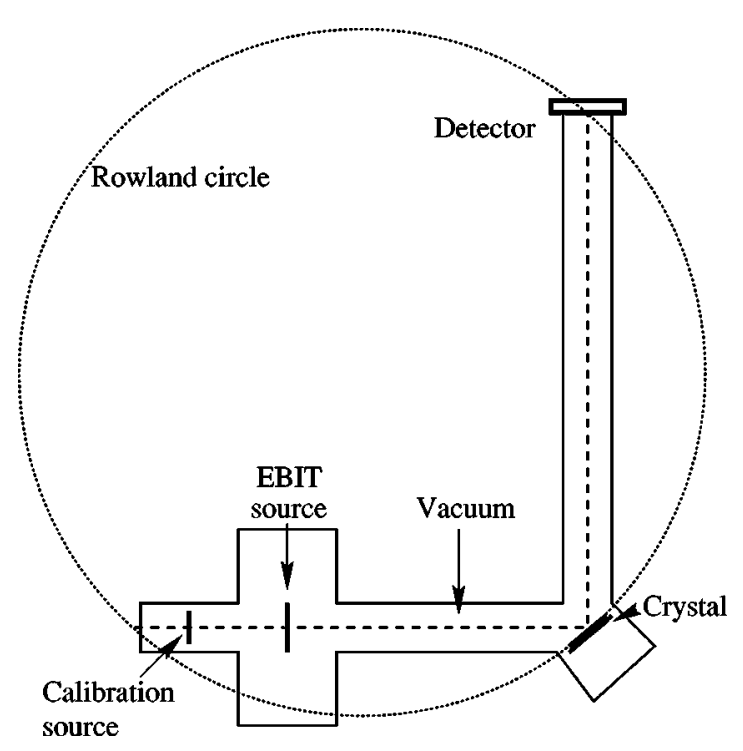

FIG. 2. Spectrometer configuration at the NIST EBIT; note that the EBIT source is located well inside the Rowland circle. The spectrometer is oriented such that the axis of the spectrometer is perpendicular to the long axis of the EBIT source and the detector arm moves vertically with changes in diffraction angle.

of $1 \mathrm{ppm}$ due to uncalculated higher-order terms. However, the discrepancy between current theories approaches $30 \mathrm{ppm}$ in the $Z=23$ region. Recent work of Persson et al. [10] estimates missing correlation effects in QED terms to be 20 ppm in the $Z=23$ region (absolute value of order $0.1 \mathrm{eV}$ across medium and high- $Z$ elements). Precise experimental determination of the heliumlike resonance lines can probe these discrepancies and uncertainties.

Most precision spectroscopy of medium- $Z$ ions has been conducted at accelerators or tokamak plasmas. The recent development of the electron-beam ion trap (EBIT) has offered another spectroscopic source to experimenters. Our experimental method takes advantage of the Doppler-free and relatively clean spectra produced by an EBIT coupled to an external calibration source to allow absolute measurements on highly charged ions. We have made absolute measurements of the heliumlike resonance lines in vanadium with an uncertainty of $27 \mathrm{ppm}$ for the $1 s 2 p^{1} P_{1} \rightarrow 1 s^{2}$ transition. Our results for vanadium do not support evidence of a systematic discrepancy between theory and experiment [11-13]. These are the first precision x-ray measurements conducted at the NIST EBIT facility.

\section{EXPERIMENT}

The experimental configuration is shown in Fig. 2. Other experiments have been conducted on the NIST EBIT using this spectrometer including polarization studies by Takács et al. [15].

The EBIT uses an intense and monoenergetic electron beam which is magnetically confined to trap and ionize charged ions $[16,17]$. Metal ion species such as vanadium are created by a metal vapor vacuum arc (MEVVA) ion source and are sequentially ionized as they enter the trap region of the electron beam. The electron-beam energy distribution of the EBIT is narrow and the energy can be tuned. The heliumlike vanadium resonance lines are excited by setting the electron-beam energy to $6.9 \mathrm{keV}$ (including space-charge correction), $1.75 \mathrm{keV}$ above the direct electron-impact excitation energy. The electron-beam current was typically 140 $\mathrm{mA}$.

We employ a Johann spectrometer with a Ge(220) crystal $(2 d=4.0007 \AA)$ which has a useful wavelength range between 1.9 and $2.8 \AA$ in first order. The radius of crystal curvature is $1.846 \mathrm{~m}$ and the resolving power $(\lambda / \Delta \lambda)$ of the spectrometer in the wavelength region of investigation is 2000-2500. The spectrometer has a Seemann wedge that can be lowered towards the crystal pole, reducing the diffracting crystal area used and the x-ray throughput but improving the resolution [18]. The detector is a two-dimensional positionsensitive proportional counter that employs capacitive charge division from a backgammon design cathode to determine position [19]. The spectrometer has a fixed crystal radius so the detector does not always remain on the Rowland circle. This has substantial systematic effects on measurements [20,21].

The spectrometer is aligned with respect to the EBIT so that the plane of crystal dispersion is parallel to the electron beam axis (see Fig. 2). The range of Bragg diffraction angles accessible with the spectrometer is $25.0-60.9^{\circ}$ and the heliumlike resonance lines of vanadium are observed at approximately $39^{\circ}$. The crystal acts as a polarizer at Bragg angles near $45^{\circ}$ and therefore radiation polarized perpendicular to the electron beam axis is the dominant diffracted component. The detector has a window wider than the full heliumlike vanadium spectrum of the EBIT. Hence several detector angles centering on various features of the spectrum were able to characterize detector linearity.

Our work is concerned with the measurement of heliumlike resonance lines. Dielectronic satellites can affect the precision of the measurements. By tuning the electron-beam energy well above the dielectronic resonance energies they are not excited, resulting in a spectrum clean of dielectronic satellite lines. Transitions from the lithiumlike states of vanadium of type $1 s 2 l 2 l^{\prime}$ lie very close to the lines of interest. Efforts must be made to minimize or eliminate these satellites from the spectra observed. The lithiumlike resonance lines that directly concern this research-due to their close location to the heliumlike resonance lines and their relatively large electron-impact cross section for inner-shell excitation-are the $q$ and $r$ [14] or $1 s 2 s 2 p^{2} P_{3 / 2}$ $\rightarrow 1 s^{2} 2 s^{2} S_{1 / 2}$ and $1 s 2 s 2 p^{2} P_{1 / 2} \rightarrow 1 s^{2} 2 s^{2} S_{1 / 2}$ transitions. Our treatment of the lithiumlike resonance lines is discussed in detail in Sec. IV.

\section{A. Calibration}

Calibration is a detailed and extensive procedure because we map the dispersion function of the spectrometer across a broad range of wavelengths around the region of interest. Using a large number of calibration lines ensures the dispersion function is well determined and a wide range of wavelengths ensures that any higher-order wavelength-dependent effects are included. We also systematically investigate the 


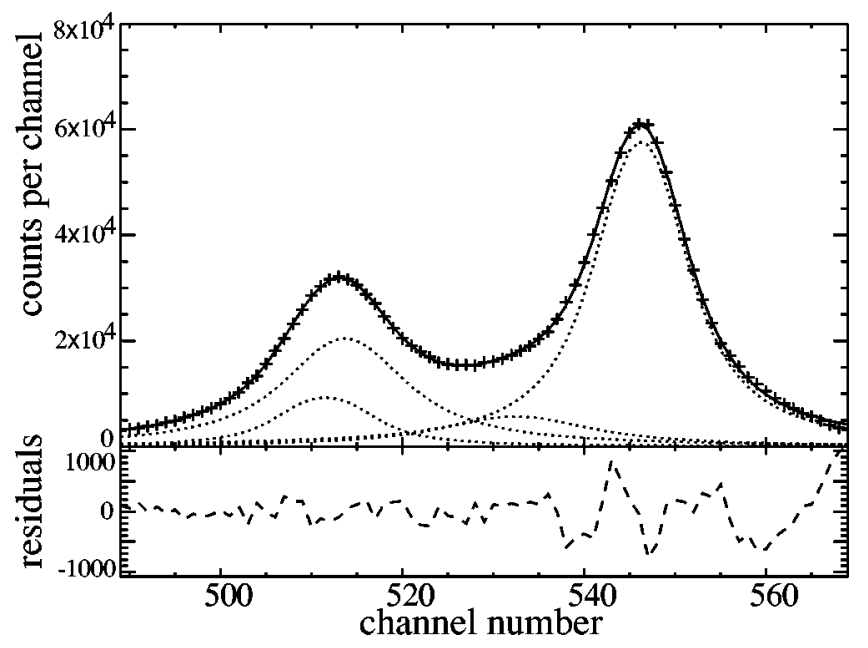

FIG. 3. Curve fitting to vanadium $K \alpha$ calibration lines. The fitted functions are Lorentzian convolved with a slit function. Four individual profiles are fitted (see text) and indicated by dotted lines. + represents data points and the solid line indicates the sum of fitted components. Residuals to the fit are shown by a dashed line underneath the main plot.

effects of variations of source size and the Seemann wedge position during calibration. Thus calibration is a time consuming but necessary procedure for an absolute precision measurement.

The calibration source consists of a $20-\mathrm{keV}$ electron gun and a series of metal targets $(\mathrm{Mn}, \mathrm{Cr}, \mathrm{V}, \mathrm{Ti})$ located on the opposite port of the EBIT to the spectrometer as indicated in Fig. 2. We locate the calibration source and the EBIT source inside the Rowland circle by design to increase flux, reduce systematic uncertainties, and increase the spectral band pass to the point that the entire vanadium spectrum can be acquired in parallel [21]. Calibration spectra are collected for a range of $K \alpha$ and $K \beta$ characteristic wavelengths (1.9-2.8 $\AA$ ) about the heliumlike resonance lines of vanadium. Bragg diffraction angles of calibration lines are in the range $29^{\circ}-45^{\circ}$ while the heliumlike resonance lines of vanadium are observed at approximately $39^{\circ} . K \alpha_{1}$ and $K \alpha_{2}$ are well resolved in our system and so the $K \alpha$ doublet provides two reference wavelengths at one detector location [20,21]. This is useful for absolute calibration of the detector channel number in micrometers. A typical vanadium $K \alpha$ calibration spectrum is shown in Fig. 3. Approximately 100 individual calibration measurements were made with some 20 independent integrated observations of heliumlike resonance lines in vanadium.

\section{B. Energy dispersive measurement}

A solid-state $\mathrm{Si}(\mathrm{Li})$ detector is used when optimizing the EBIT conditions to maximize the flux of heliumlike vanadium transitions and to minimize contamination from other charge states and dielectronic recombination transitions. An example of a typical $\mathrm{Si}(\mathrm{Li})$ spectrum is shown in Fig. 4. The resolution is sufficient to identify hydrogenic and heliumlike vanadium as well as various barium charge states (barium, emanating from the electron gun, is common in EBIT spec-

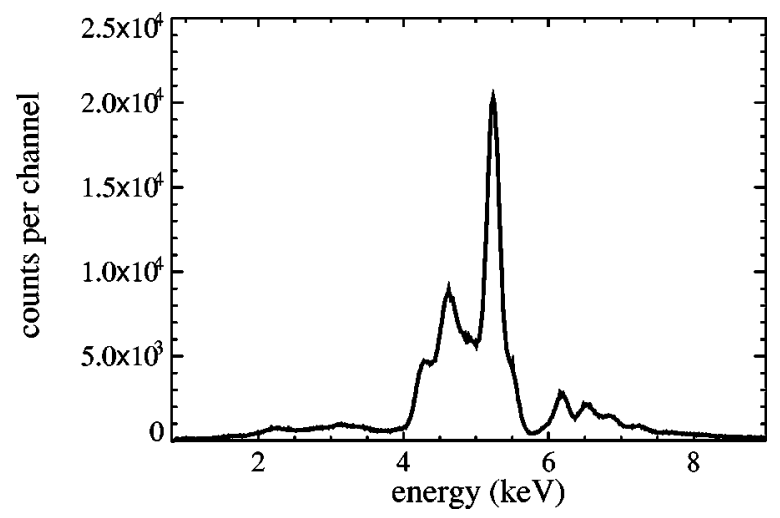

FIG. 4. General features of the $\mathrm{Si}(\mathrm{Li})$ spectrum observed on the NIST EBIT: The highest peak at $5.2 \mathrm{keV}$ is composed of a blend of the heliumlike vanadium resonance lines, and the broad peak between $4.6-4.9 \mathrm{keV}$ is predominantly neonlike barium and other barium charge states.

tra). A small amount of pure nitrogen gas is leaked into the trap to increase the proportion of higher charge states via evaporative cooling.

In the EBIT an axial trapping potential is created by a series of three drift tubes. The voltage applied to the middle drift tube determines the energy of the electron beam and sets the position of the trap with respect to the ground potential. The drift tube voltages can be switched rapidly in a time sequence. This is necessary in order to catch the ions injected from the MEVVA ion source and to empty the trap after a certain amount of time to avoid the accumulation of unwanted long lifetime charge states and background barium ions. The time sequencing was optimized using the signal from the $\mathrm{Si}(\mathrm{Li})$ detector.

Once the optimum EBIT operating conditions are chosen, crystal spectrometer data are collected while continuing to monitor the performance with the $\mathrm{Si}(\mathrm{Li})$ detector. A single spectrum is collected and saved for each period, approximately $2 \mathrm{~h}$, between liquid nitrogen fills of the outer cryogenic jacket of the EBIT. Spectra are collected continuously for several days with a 24-hour-a-day EBIT operation. Spectra collected under the same conditions are then summed during the analysis of data. Figure 5 shows a heliumlike vanadium spectrum from $24 \mathrm{~h}$ of observation with the crystal spectrometer. The position and relative intensity of the $q$ lithiumlike resonance line is also indicated in Fig. 5. Contamination from the $r$ line is negligible.

\section{Clinometry}

During the relatively long data acquisition periods, care must be taken to monitor and minimize any shifts in the diffraction angle or detector position. Clinometers continually monitor the detector and crystal angles with arcsecond resolution and can be used to determine any changes in angular position during EBIT observations. The clinometers also record certain large amplitude vibrations of the detector arm and crystal mount. Such vibrations may be caused by faults in vacuum pumps or rapid surging of liquid nitrogen through the feed lines to the EBIT. Data obtained displaying these vibrations are rejected because the diffraction profiles 


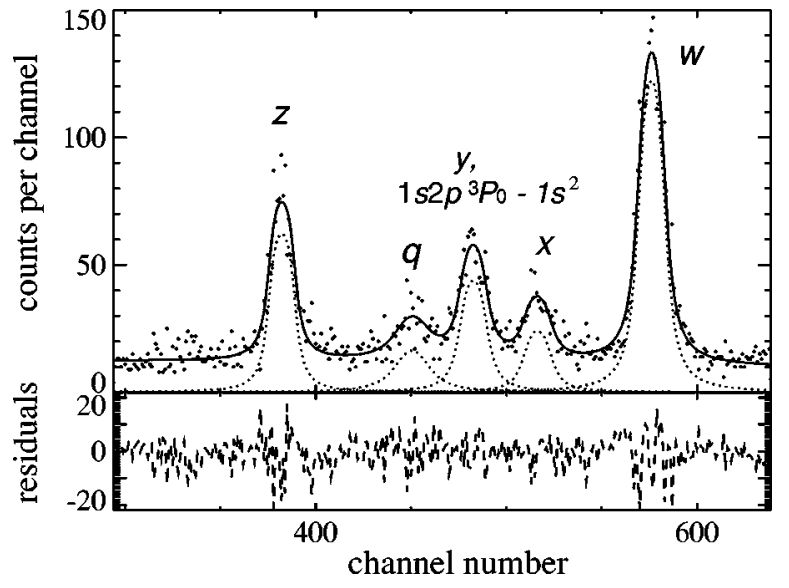

FIG. 5. Heliumlike vanadium spectra observed at the NIST EBIT. Resonance lines and lithiumlike satellite lines are labeled according to Gabriel's scheme. Summed observations represent some 24 of observation. The $q\left(1 s 2 s 2 p^{2} P_{3 / 2} \rightarrow 1 s^{2} 2 s^{2} S_{1 / 2}\right)$ lithiumlike satellite contamination is at a level which does not impair the final measurement. Spectra are fitted with a Lorentzian convolved with a slit profile and a background. The dotted lines are the fitted profiles to each resonance line; the solid line is the sum of all fitted profiles and background.

will be broadened, compromising resolution and precision. Rejected data comprised a small subset of the total measurements. Most observations were made with the Seemann wedge removed to maximize flux.

A post-measurement calibration was conducted to insure that no changes in the spectroscopy system had occurred during the measurement process. The postrun calibration, when subsequently analyzed in conjunction with prerun calibration data, was used to ensure against physical shifts and changes in alignment.

\section{CURVED CRYSTAL THEORY AND SYSTEMATIC SHIFTS}

Curved crystal geometries are often used in precision $\mathrm{X}$-ray spectroscopy to obtain increased signal and lower statistical error, or to focus an extended source. All systematic shifts down to the level of 10-30 ppm must be accounted for to perform useful tests of QED in medium $Z$ highly charged ions. Dynamical $x$-ray diffraction plays a crucial role in any wavelength dispersive spectroscopy such as ours. A systematic shift which is commonly accounted for in X-ray diffraction is the refractive index $(\mathrm{RI})$ correction which is due to the mean change in refractive index in the crystal changing the angle of incidence relative to the air or vacuum. The angle of diffraction $\theta_{B}$ is shifted causing Bragg's law of diffraction

$$
n \lambda=2 d \sin \theta_{B}
$$

to become

$$
n \lambda=2 d \sin \theta_{B}\left(1-\frac{1-R I}{\sin ^{2} \theta_{B}}\right)
$$

where RI is the index of refraction (specific to $\lambda$ and the crystal). The magnitude of the refractive index correction is 100-300 ppm [22,23], depending upon the wavelength, crystal, and geometry, and so must be determined to a few percent.

Most curved and flat crystal spectroscopy QED investigations have addressed systematic corrections based upon raytracing methods, incorporating the simple refractive index correction [24-26]. Some of these QED measurements were calibrated using wavelengths observed in different orders of diffraction [11]. These measurements, however, did not use a full dynamical diffraction theory to calculate systematic shifts and therefore did not consider significant effects relating to depth penetration, lateral shifts, source size, and detector location. Any of these effects can cause systematic shifts comparable to the refractive index correction.

\section{A. Depth penetration}

Depth penetration refers to the mean depth that $\mathrm{x}$-rays penetrate into the crystal and the effect that this penetration has upon the observed diffraction angle. For curved crystals depth penetration at angles other than normal to the surface will result in a variation of the angle of incidence in successively deeper crystal layers. The depth penetration of x-rays into a crystal can have effects larger than refractive index shifts [23]. It is therefore the dominant systematic in many curved crystal measurements, and is independent of refractive index and ray-tracing (geometrical) corrections. The magnitude of the depth penetration is dependent upon diffraction order and angle of incidence. The resulting shift in angle for curved crystals (radius $2 R_{z}$ where $R_{z}$ is the Rowland circle radius) can be estimated using

$$
\Delta \theta_{\mathrm{in} / \mathrm{out}} \simeq \arccos \left[\left(1+\frac{\bar{d}}{2 R_{z}}\right) \cos \left(\theta_{B} \pm \alpha_{p}\right)\right]-\left(\theta_{B} \pm \alpha_{p}\right),
$$

where $\bar{d}$ is the mean depth of penetration and $\alpha_{p}$ is the mean angle of diffracting planes to the crystal surface [23]. Simple estimates of $\bar{d}$ can be made but a rigorous calculation with $1 \%$ precision requires the dynamical diffraction theory. The strong dependence upon diffraction order, due to the dependence of attenuation $(\mu)$ upon energy, implies that any x-ray QED measurement employing diffraction in different orders should address this effect explicitly.

\section{B. Lateral shifts}

Lateral shifts are also due to depth penetration and refer to a shift in the exit location of an x-ray at the crystal surface. For flat crystals this results in a transverse shift at the detector and is dependent on $\mu$ and extinction. For curved crystals the lateral shift causes a shift at the detector (if positioned on the Rowland circle) of

$$
\Delta Y_{\text {lateral }}(A, B) \simeq R_{z} \arccos [\Phi]-2 R_{z} B
$$

where 


$$
\begin{aligned}
\Phi= & {[(2 \cos B-\cos A) \cos A} \\
& \left.-\sqrt{\sin ^{2} A\left(\sin ^{2} A+\cos ^{2} B-\cos B \cos A\right)}\right]
\end{aligned}
$$

and $B=\theta_{B}+\alpha_{p}, A=B+\theta_{A}$, and $\theta_{A}=\Delta \theta_{\text {in }}+\Delta \theta_{\text {out }}$ as given by Eq. (3). Simpler, less precise estimates can be formulated for specific situations but ultimately any calculation relies on a precise determination of depth penetration. For low angle and low order diffraction, lateral shifts will be $10-100 \%$ of the refractive index corrections [23]. Equation (4) will determine the lateral shift on the Rowland circle but not the shift at a detector located off the Rowland circle.

\section{Source and detector shape and position}

Geometrical effects such as source size and position can cause shifts of a similar magnitude to refraction [23]. While ray tracing can adequately describe these situations outside the crystal, the effect of dynamical diffraction, associated depth penetration, and lateral shifts modifies these estimates.

Estimates of shifts or errors presented in curved crystal geometries are often calculated for an ideal detector located on the Rowland circle. However, the detection surface is usually flat and therefore does not conform to the Rowland circle. Detectors located on a fixed length detector arm will also travel off the Rowland circle with a variation in the detection angle unless the crystal curvature is also varied precisely.

Mosaicity and crystal curvature effects are also often disregarded but these effects must be considered for the precision which has been the aim of this research. These issues led to the development of the theory that combines curvature and mosaicity in a dynamical diffraction theory by Chantler $[27,28]$. One systematic shift is caused by the calibration source not being in the same location as the EBIT source. Theoretical modeling determines the shifts associated with this mislocation and the correction is $3-5 \mathrm{ppm}$ depending upon wavelength.

Detailed theoretical modeling of our spectroscopy system has revealed a wavelength-dependent systematic error associated with detector shape and location due to asymmetric partial focusing on the detector plate. Conventional shifts calculated for detection on the Rowland circle do not agree with shifts computed for flat extended detectors. This systematic error can be 100-200 ppm and would be present in any Johann curved crystal spectrometer with fixed length detector arm and flat detection surface.

In later work [20,21] this theory was expanded to incorporate flat surface detectors located off the Rowland circle. We have therefore developed the computational techniques to deal with all of the above-mentioned systematics in a rigorous and integrated manner.

\section{DATA ANALYSIS AND ERROR SOURCES}

Calibration and EBIT spectra are fitted with Lorentzian line shapes convolved with slit profiles. The width of the Lorentzian and common slit components are free parameters in the fit. The background is negligible and is not fitted. For calibration lines, two components are fitted to each line fol- lowing Deutsch et al. [29] and Hartwig et al. [30] to reproduce the asymmetry of real profiles (see Fig. 3). The heliumlike resonance lines and satellite contamination are fitted by a single component with a Lorentzian convolved with a slit profile in addition to a quadratic background. Figure 5 shows the result of profile fitting to a heliumlike vanadium spectrum.

The location of the calibration lines with two fitted components is determined from the peak position of the sum of the two components. Peak values are used to correspond to the reference values of Bearden [22] (this point is discussed further in Sec. IV B). For the heliumlike resonance lines the centroid position and peak position correspond since the line shapes are symmetrical and only one component is fitted to each line.

\section{A. Dispersion function determination}

Bearden's tabulation of characteristic x-ray lines [22], although published in 1967, is the most comprehensive data set available. Reference wavelengths for calibration lines are corrected from Bearden's values using the recent CODATA determination of lattice spacings [31]. The adjustment required is relatively constant at $-15 \mathrm{ppm}$ across the wavelengths we use for calibration. The dispersion function is fitted to the 10 calibration wavelengths: Ti $K \beta_{1,3}, \mathrm{~V} K \alpha_{1}, \mathrm{~V}$ $K \alpha_{2}$, V $K \beta_{1,3}$, Cr $K \alpha_{1}$, Cr $K \alpha_{2}$, Cr $K \beta_{1,3}$, Mn $K \alpha_{1}$, Mn $K \alpha_{2}$, and $\mathrm{Mn} K \beta_{1,3}$. Prerun and postrun calibration data can be compared for consistency and systematic shifts. The dispersion function relates the wavelength of a spectral feature located at the detector center to the angle of diffraction which is measured by clinometers attached to crystal and detector arms of the spectrometer.

Clinometer values for the crystal and detector are continually recorded with any spectrum and the centroid of each clinometer distribution is the measure of the angle for crystal $\left(\theta_{B}\right)$ and the detector $\left(2 \theta_{B}\right)$. The dispersion function is determined by a bivariate nonlinear least-squares fit to the function

$$
\Theta=\arcsin \left[\frac{D C-A(1)}{A(2)}\right]-\arcsin \left[\frac{C C-A(3)}{A(4)}\right]+A(0),
$$

where $D C$ is the centroid of the detector clinometer, $C C$ the centroid of the crystal clinometer, $\Theta$ the reference wavelength of the calibration line (adjusted for refractive index correction and other systematic shifts), and $A(0)-A(4)$ the parameters of the fit relating to offsets and scaling of the clinometer response function, which has been demonstrated to be highly linear in $\sin \theta$.

We observe all the resonance lines of heliumlike vanadium at one spectrometer setting. Thus the position-sensitive detector scale (in channels/ $\mu \mathrm{m}$ or channels/arcsecond) is an important input into the dispersion function.

Any systematic shifts must be accounted for when determining the dispersion function. As described in the preceding section, we account for refractive index corrections, depth penetration of $x$ rays in the crystal, shifts associated with source size and location, and shifts due to detector 
TABLE I. Error budget for heliumlike vanadium measurement.

\begin{tabular}{|c|c|c|c|c|}
\hline \multirow[b]{2}{*}{ Error source } & \multicolumn{4}{|c|}{$\begin{array}{l}\text { Magnitude of error (ppm) } \\
\text { Resonance line }\end{array}$} \\
\hline & $w$ & $x$ & $y$ & $z$ \\
\hline Statistical uncertainty of centroid position & 9.5 & 31.0 & 22.3 & 12.9 \\
\hline Reference wavelengths (Bearden [22]) & & & 12 & \\
\hline Adjustment of Bearden wavelengths & & & $<0.5$ & \\
\hline Diffraction theory ${ }^{\mathrm{a}}$ & & & 6 & \\
\hline Dispersion function determination $^{\mathrm{a}}$ & & & 20 & \\
\hline Temperature and diffraction crystal $2 d$ spacing variation & & & $<5$ & \\
\hline Doppler shifts & & & $<4$ & \\
\hline Total excluding statistics ${ }^{\mathrm{b}}$ & & & 24.9 & \\
\hline Total & 26.7 & 39.8 & 33.4 & 28.1 \\
\hline
\end{tabular}

${ }^{\mathrm{a}}$ Summation of related uncertainties (see text).

${ }^{b}$ Total excluding statistics: Sum of all errors except statistical uncertainty of heliumlike vanadium observations.

shape and location. The dispersion function is not a simple relationship between angle and wavelength but a complex (but smooth) function of reference wavelengths, clinometer values, detector scale, and systematic shifts.

\section{B. Error budget}

The error budget is laid out in Table I. The statistical uncertainty varies between $9.5 \mathrm{ppm}\left(w, 1 s 2 p{ }^{1} P_{1} \rightarrow 1 s^{2}\right)$ and $31.0 \mathrm{ppm}\left(x, 1 s 2 p^{3} P_{2} \rightarrow 1 s^{2}\right)$ with the relatively low flux of the EBIT prohibiting trivial improvement. The uncertainty is determined from the curve fitting routine and is the standard deviation of the centroid position for each resonance. This corresponds to a determination varying between $1 / 27$ and $1 / 77$ of the widths.

Recent work by Deslattes et al. [32] on reference wavelengths has resulted in lower estimates of uncertainties than those on Bearden's values. Bearden's uncertainties in general have been reduced by $25-30 \%$ from $12 \mathrm{ppm}$ to $8-10 \mathrm{ppm}$. In most cases, including all the wavelengths used in our investigations, the further refinement yielded the same value for the wavelengths within uncertainties. Our values (adjusted from Bearden) and those of Deslattes et al. have a small discrepancy of $0.5 \mathrm{ppm}$. This discrepancy is insignificant compared to the uncertainty of the wavelengths and does not compromise our final result.

The peak locations in the spectra and the derived centroid determinations for $\mathrm{V} K \alpha_{1}, \mathrm{~V} K \alpha_{2}$ differ by 50-80 ppm. The choice of peak and centroid values in our calibration system is an important systematic effect. We have found that the shift does not create an error in first order if theory and experimental results are computed consistently for peak or centroid values. Peak values for single-vacancy spectra remain the preferred measure for reporting or determining characteristic energies despite advanced methods for determining centroids [33]. In the final analysis peak values are therefore used and determined from curve fitting to experimental profiles as discussed in Sec. IV.

Diffraction theory uncertainty refers to the diffraction calculations that are used to determine systematic shifts. The reliability and self-consistency of these calculations when compared to calibration lines resulted in a variance of less than $6 \mathrm{ppm}$. The magnitude of each of the component shifts are of order $100 \mathrm{ppm}$ (and vary quite dramatically for the range of angles and energies of interest), but the uncertainty on the net result is $6 \mathrm{ppm}$. We emphasize that $6 \mathrm{ppm}$ is not the net shift which varies for our experiment from about 70 to $300 \mathrm{ppm}$. The uncertainty is low for the total calculated shift because of relative insensitivity of the result to experimental uncertainties in source location, source size, and detector location. To detail one example, the shift associated with the mislocation of the calibration and EBIT sources results in a small shift (3-5 ppm); the separation of these sources is $0.25 \mathrm{~m}$. It follows that uncertainty in the longitudinal position of the sources at the millimeter level is not critical.

The uncertainty of the dispersion function determination shown in Table I is currently the dominant systematic correction. The determination of the dispersion function involves interrelated variables, and the absolute uncertainties of these input variables do not add linearly to the final uncertainty of the function. For example, the calibration source size uncertainty is $10 \%$ but in the Johann geometry this contributes less than $5 \mathrm{ppm}$ to wavelength determination. Longitudinal source location is very uncertain $( \pm 1 \mathrm{~mm})$ but in our geometry this has a negligible effect on the final accuracy. The overall contribution of calibration source size and alignment uncertainty is $5 \mathrm{ppm}$. The statistical error associated with the calibration lines is $2-3 \mathrm{ppm}$ and the error associated with the calibration profile fitting is 3-4 ppm.

The major contribution to the dispersion function uncertainty is the detector scale uncertainty. This uncertainty relates to the absolute calibration of the detector position scale in channels per micrometer and the linearity of that scale. The heliumlike resonance lines are widely spaced on our detector (" $w$ " - " $z$ " separation $\approx 190$ channels or $\approx 8.5 \mathrm{~mm}$ ) and therefore any uncertainty in the detector scale is critical. The absolute detector scale is determined at less than $0.5 \%$ which results in an average contribution to the dispersion 
TABLE II. Energies of heliumlike resonance lines in vanadium, comparison to theory.

\begin{tabular}{|c|c|c|c|c|c|c|}
\hline \multirow[b]{2}{*}{ Notation } & \multirow[b]{2}{*}{ Transition } & \multirow{2}{*}{$\begin{array}{c}\text { Theory } \\
\text { Energy } \\
(\mathrm{eV})\end{array}$} & \multirow[b]{2}{*}{$\begin{array}{l}\text { QED cont. } \\
\text { (ppm) }\end{array}$} & \multicolumn{3}{|c|}{ NIST EBIT and Univ. Melbourne (this work) } \\
\hline & & & & $\begin{array}{c}\text { Energy } \\
(\mathrm{eV})\end{array}$ & $\begin{array}{c}\Delta E^{\mathrm{c}} \\
(\mathrm{ppm})\end{array}$ & $\begin{array}{c}\text { Expt. test of QED } \\
(\%)\end{array}$ \\
\hline$w$ & $1 s 2 p{ }^{1} P_{1} \rightarrow 1 s^{2}{ }^{1} S_{0}$ & 5205.15 & 471.1 & $5205.10(14)$ & $-10(27)$ & $5.7 \%$ \\
\hline$x$ & $1 s 2 p{ }^{3} P_{2} \rightarrow 1 s^{2}{ }^{1} S_{0}$ & 5188.72 & 478.1 & $5189.12(21)$ & $80(40)$ & $8.4 \%$ \\
\hline$y$ & $1 s 2 p{ }^{3} P_{1} \rightarrow 1 s^{2}{ }^{1} S_{0}$ & 5180.31 & 482.5 & $5180.22(17)^{\mathrm{d}}$ & $-19(33)$ & $6.9 \%$ \\
\hline$z$ & $1 s 2 s^{3} S_{1} \rightarrow 1 s^{2}{ }^{1} S_{0}$ & 5153.88 & 415.5 & $5153.82(14)$ & $-11(28)$ & $6.7 \%$ \\
\hline
\end{tabular}

arake (Ref. [5])

${ }^{\mathrm{b}} \mathrm{QED}$ cont.: QED contribution to transition energy using QED definitions of Drake [5].

${ }^{\mathrm{c}} \Delta E$ : Energy (expt.-theory)/theory.

${ }^{\mathrm{d}}$ Blend of $y$ and $1 s 2 p^{3} P_{0} \rightarrow 1 s^{2}$ transitions.

function imprecision of $15 \mathrm{ppm}$.

The contribution of satellite lines has also been included in the dispersion function uncertainty. The $q\left(1 s 2 s 2 p^{2} P_{3 / 2}\right.$ $\rightarrow 1 s^{2} 2 s^{2} S_{1 / 2}$ ) lithiumlike satellite is explicitly fitted (see Fig. 5) and has a peak intensity of $13 \%$ of the $w$ intensity. Any dielectronic satellites will be at least an order of magnitude less intense than the lithiumlike satellites or less than $1 \%$ of the intensity of the heliumlike resonance lines [with full widths at half maximums (FWHMs) corresponding to 600-800 ppm of the resonance energy]. Thus any unresolved satellite, even located at a half-width from the centroid of a heliumlike resonance line, will cause a shift of no more than 3-4 ppm.

Dispersion function uncertainty has been reduced to 20 ppm through the careful determination of systematic shifts. This has been a major achievement of this work. We have done explicit analysis to determine the error associated with the omission of the systematic shift caused by a flat detector shape and location off the Rowland circle. This revealed a poor determination of the dispersion function and errors of $100 \mathrm{ppm}$, which was corrected for as described above. Further improvement with future experiments is expected.

Doppler shifts due to the low thermal velocities of the highly charged ions in the EBIT are not significant sources of uncertainty. On average the Doppler shifts will be zero as there is no preferred direction of motion. Doppler broadening is $1.8 \mathrm{eV}$ for $1 \mathrm{keV}$ ions and we allow for a possible $1 \%$ asymmetry of velocity distribution resulting in a maximum Doppler shift of less than $0.02 \mathrm{eV}$ or less than $4 \mathrm{ppm}$. Temperature effects, particularly on the diffraction crystal $2 \mathrm{~d}$ spacing, are also insignificant sources of uncertainty. The linear expansion coefficient of the germanium crystal we use is $5.95 \times 10^{-6} \mathrm{~K}^{-1}$ [34] and the crystal is in moderate vacuum $\left(10^{-6}\right.$ Torr $)$ with monitored external temperature variations of $\simeq 2{ }^{\circ} \mathrm{C}$. Also any direct lattice spacing errors have only a second-order effect on the measurement accuracy (because of the calibration) so that the effect on wavelength determination will be less than $5 \mathrm{ppm}$.

Summing all systematics errors in quadrature results in a $24.9 \mathrm{ppm}$ systematic uncertainty and a total uncertainty for the resonance lines of 27-40 ppm. The main sources of uncertainty are therefore statistical, reference wavelengths, and dispersion function determination. All may be regarded as soft limits at present (subject to improvement with additional work in the future). Methods of reducing statistical uncertainty by improving spectrometer efficiency are being investigated and improved flux from the NIST EBIT has been achieved in subsequent studies. Reference wavelength uncertainty is limited at present to Bearden's values but some of the metals we employ for calibration have been measured with improved precision recently [35]. Future measurements of the characteristic wavelengths on, for example, a vacuum double-crystal spectrometer are very likely to reduce uncertainty.

\section{RESULTS AND DISCUSSION}

Our results are the first absolute measurements of all the resonance lines in heliumlike vanadium using an EBIT. We do not rely on a single calibration energy, but require a series of calibration lines to determine the dispersion function of the spectrometer. These measurements represent a $27-40$ ppm determination of the heliumlike resonance lines in vanadium. Results are summarized in Table II and the notation of Gabriel [14] for each transition is indicated.

\section{A. Comparison to Theory}

Our results for all of the heliumlike vanadium resonance lines are compared to the theory of Drake [5] in Table II. The values for the $w\left(1 s 2 s^{1} P_{1} \rightarrow 1 s^{2}\right)$ and $z\left(1 s 2 s^{3} S_{1} \rightarrow 1 s^{2}\right)$ transitions lie just below theory but well within experimental uncertainty. The $x\left(1 s 2 p^{3} P_{2} \rightarrow 1 s^{2}\right)$ transition is the least intense of the heliumlike resonance lines, so the statistical uncertainty is larger. However, the result for the $x$ line is less than $2 \sigma$ from theory [5] which is within reasonable statistical variation. The comparison of the $y\left(1 s 2{ }^{3} P_{1} \rightarrow 1 s^{2}\right)$ and $1 s 2{ }^{3} P_{0} \rightarrow 1 s^{2}$ blend to theory is discussed in detail in Sec. VF.

The uncertainty of theoretical calculations including the estimation of missing or uncalculated terms has been receiving increasing scrutiny as techniques have advanced. One of the most recent two-electron Lamb shift calculations by Persson et al. [10] estimates missing correlation effects in QED contributions at $0.1 \mathrm{eV}$ for all elements or $20 \mathrm{ppm}$ of transition energies in medium $Z$ ions. In earlier work, Drake [5] 
TABLE III. Selected $w\left(1 s 2{ }^{1} P_{1} \rightarrow 1 s^{2}\right)$ transition energies $(\mathrm{eV})$ for vanadium and surrounding medium $Z$ ions.

\begin{tabular}{|c|c|c|c|c|c|c|c|c|c|}
\hline \multirow[b]{2}{*}{$Z$} & \multirow[b]{2}{*}{ Experiment } & \multirow[b]{2}{*}{ Ref. } & \multicolumn{4}{|c|}{ Theoretical transition energies } & \multirow{2}{*}{$\begin{array}{l}\Delta \mathrm{Th}^{\mathrm{a}} \\
(\mathrm{ppm})\end{array}$} & \multirow{2}{*}{$\begin{array}{l}\text { QED }^{\mathrm{b}} \\
(\mathrm{eV})\end{array}$} & \multirow{2}{*}{$\begin{array}{c}2 e \mathrm{QED}^{\mathrm{c}} \\
(\mathrm{eV})\end{array}$} \\
\hline & & & Unified [5] & $\mathrm{AO}[6]$ & CI [7] & $\operatorname{MCDF}[9]$ & & & \\
\hline 18 & $3139.553(38)$ & {$[47]$} & 3139.577 & 3139.582 & 3139.617 & 3139.65 & 23 & 1.055 & 0.09 \\
\hline 22 & 4749.74(17) & {$[11]$} & 4749.63 & 4749.64 & 4749.71 & & 17 & & \\
\hline 23 & $5205.10(14)$ & {$\left[{ }^{\mathrm{d}}\right]$} & 5205.15 & 5205.16 & & & & 2.474 & 0.16 \\
\hline 24 & $5682.32(40)$ & {$[11]$} & 5682.05 & 5682.06 & 5682.15 & & 18 & & \\
\hline 26 & $6700.08(24)$ & {$[27]$} & 6700.40 & 6700.43 & 6700.54 & 6700.60 & 30 & & \\
\hline 32 & $10280.70(22)$ & {$[13]$} & 10280.14 & 10280.19 & 10280.39 & & 24 & 7.674 & 0.40 \\
\hline
\end{tabular}

${ }^{\mathrm{a}} \Delta \mathrm{Th}$ : Maximum discrepancy between theories.

${ }^{\mathrm{b}} \mathrm{QED}$ : QED contribution to the ground state $\left(1 s^{2}{ }^{1} S_{0}\right)$ [5].

c $2 e$ QED: Two-electron QED contribution extrapolated (see text) [10].

${ }^{\mathrm{d}}$ This work.

claimed uncertainty for $Z=23$ was less than $0.005 \mathrm{eV}$ or 1 ppm of heliumlike resonance lines due to uncalculated higher-order terms. Some of the latest theoretical calculations for the $w$ transition in medium $Z$ ions are summarized in Table III. The discrepancy between theories is indicated and the maximum discrepancy ranges from 23 to $30 \mathrm{ppm}$ for $Z=18-26$ and differences are consistent. Our measurements are at this level of uncertainty.

Table II also shows the QED contributions to heliumlike resonance lines in vanadium as determined by Drake. The QED contributions are also expressed as a proportion of the relevant transition in ppm. The level at which our measurements test these contributions is between $5.7 \%$ and $8 \%$. The theoretical QED contributions include mass polarization and nuclear size effects but these contribute less than $1 \%$ to the total. If the QED contributions to the $2 l$ states are assumed to be correct, then the $1 s$ QED contribution is measured to $6 \%$.

Our result for the $w$ line in vanadium is within our experimental uncertainty of the theory of Drake [5] and Plante et al. [6]. We therefore find no evidence of the earlier reported trend that experimental values are greater than theory [11]. Figure 6 shows our result for the $w$ transition compared to theory [5] and other measurements for heliumlike vanadium and surrounding medium $Z$ heliumlike ions. Our measurements are compared with other experiments in the context of medium $Z$ x-ray measurements in the following discussion.

\section{B. Two-electron Lamb shifts}

The two-electron Lamb shift has been a topic of considerable interest recently following significant variation in the recent calculations of two-electron contributions to the ground state of medium and high $Z$ heliumlike ions [10,6,5]. The "two-electron Lamb shift" can be defined as the difference between the hydrogenic $1 s_{1 / 2}$ Lamb shift and the QED contributions to the heliumlike ground state $\left(1 s^{2}{ }^{1} S_{0}\right)$. The two-electron Lamb shift relates to the two-electron radiative QED Feynman diagrams (vacuum polarization and selfenergy) due to interactions between the electrons [10]. Nonradiative two-electron QED (ladder and crossed-photon diagrams) are negligible for $Z \leqslant 32$. We have calculated the twoelectron Lamb shift by subtracting the results of Johnson and
Soff [37] for the hydrogenic $1 s_{1 / 2}$ Lamb shift from the QED contributions to the heliumlike ground state from Drake [5]. For $Z=32$ (germanium) we obtain $0.31 \mathrm{eV}$ which can be directly compared to the $0.4 \mathrm{eV}$ result of Persson et al. [10]. For vanadium, the two-electron Lamb shift calculated in this manner is $0.15 \mathrm{eV}$ or $29 \mathrm{ppm}$ of the $1 s 2 s^{1} P_{1} \rightarrow 1 s^{2}$ transition energy. Two-electron QED calculations for $Z<32$ are not available from Persson et al., so no direct comparison can be made with Drake's calculation of vanadium.

As a simple alternative calculation, we have estimated the two-electron Lamb shift for heliumlike ions of $Z$ less than 32 by extrapolating the results of Persson et al. for $Z=32-92$ using a power-law fit $\left(2 e \mathrm{QED}=a Z^{b}\right)$. The $Z$ power dependence is interestingly found to be $b \approx 2.5$. Derived results for $Z=18$ and 23 are tabulated with Persson's result for $Z=32$ in Table III. Theory might expect a power-law dependence of $b=3$ as the two-electron contributions scale as $Z^{3}$. The result ( $b \approx 2.5$ ) from the power-law fit to the sum of all computed contributions is in reasonable agreement with the expected theoretical value. For vanadium the two-electron Lamb shift is $0.16 \mathrm{eV}$ or $31 \mathrm{ppm}$, slightly larger than the

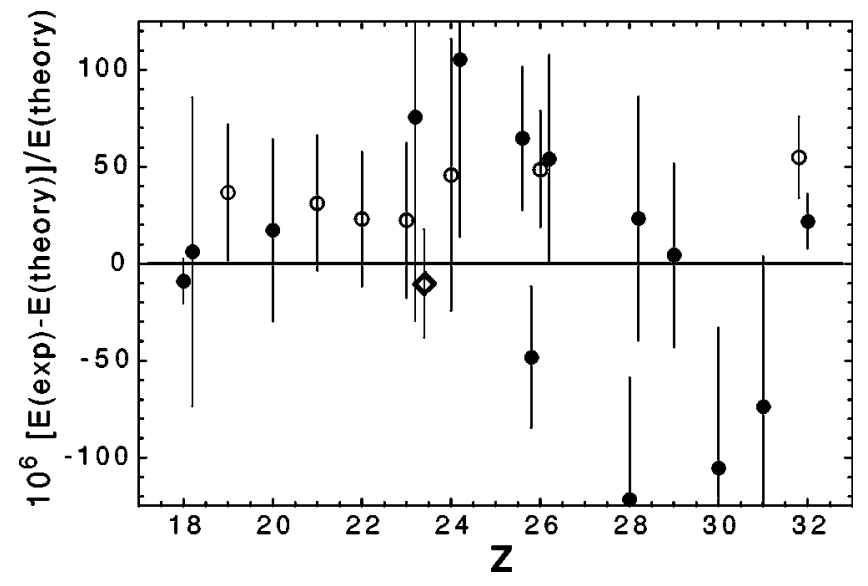

FIG. 6. Measurements of the $w\left(1 s 2{ }^{1} P_{1} \rightarrow 1 s^{2}\right)$ transition in medium $Z$ ions: comparison to theoretical work of Drake [5]. $E(\exp )$ : experimental transition energy; $E$ (theory): theoretical transition energy. $\diamond$, this work; $\bigcirc$, Beiersdorfer et al. (1989), Ref. [11], $Z=32$, MacLaren et al. (1992), Ref. [13]; @, other sources (see Table III references). 
TABLE IV. Experimentally determined energies (eV) of heliumlike vanadium $1 s 2 l \rightarrow 1 s^{2}$ resonance lines. The source of highly charged ions is indicated.

\begin{tabular}{|c|c|c|c|c|c|c|c|c|}
\hline \multirow[b]{2}{*}{ Key } & \multirow[b]{2}{*}{ Transition } & \multirow{2}{*}{$\begin{array}{l}\text { PLT tokamak } \\
\quad \text { Ref. [11] }\end{array}$} & \multirow{2}{*}{$\begin{array}{l}\text { LIVS plasma } \\
\text { Ref. [39] }\end{array}$} & \multirow[b]{2}{*}{ Energy } & \multicolumn{2}{|c|}{ LLNL EBIT [12] } & \multicolumn{2}{|c|}{ TFR tokamak [38] } \\
\hline & & & & & $E_{w}-E^{\mathrm{a}}$ & $d E^{\mathrm{b}}$ & $E_{w}-E$ & $d E$ \\
\hline$w$ & $1 s 2 p{ }^{1} P_{1} \rightarrow 1 s^{2}$ & $5205.27(21)$ & $5205.58(55)$ & $5205.33^{\mathrm{c}}$ & & & & \\
\hline$x$ & $1 s 2 p^{3} P_{2} \rightarrow 1 s^{2}$ & & & $5188.86(22)$ & 16.47 & 0.04 & 17.0 & 0.56 \\
\hline$y$ & $1 s 2 p^{3} P_{1} \rightarrow 1 s^{2}$ & & & $5180.30(22)$ & 25.03 & 0.19 & 25.7 & 0.82 \\
\hline${ }^{3} P_{0}$ & $1 s 2 p^{3} P_{0} \rightarrow 1 s^{2}$ & & & $5180.30(22)$ & 25.03 & -1.28 & 25.7 & -0.65 \\
\hline$z$ & $1 s 2 s^{3} S_{1} \rightarrow 1 s^{2}$ & & & $5153.90(30)$ & 51.43 & 0.16 & 51.9 & 0.66 \\
\hline
\end{tabular}

${ }^{\mathrm{a}} E_{w}-E$ : difference between energy of $w$ transition and energy of relevant transition.

${ }^{\mathrm{b}} d E=\left(E_{w}-E\right)_{\text {theory }}-\left(E_{w}-E\right)_{\text {expt }}$.

${ }^{\mathrm{c}}$ Reference line set to semiempirical value of $5205.33 \mathrm{eV}$.

experimental uncertainty of $0.14 \mathrm{eV}$ of our measurements. The agreement between this result obtained from extrapolation of the results of Persson et al. and the value obtained by direct calculation from Johnson and Soff and Drake is excellent ( $0.01 \mathrm{eV}$ difference). For $Z=32$, where the comparison can be made directly without extrapolation, the difference is $0.09 \mathrm{eV}$, remarkably consistent with the uncertainty estimate $(0.1 \mathrm{eV})$ of Persson et al. for contributions from missing correlation effects in QED. As was the case with the experiment of Marrs et al. [36], a significant improvement in experimental precision would provide a critical test of two-electron QED.

In terms of basic physical effects included, the calculations of Drake and of Persson et al. are equivalent up to all terms of order $\alpha^{3}$ (assuming that the many-body perturbation theory expansion has converged sufficiently well), and also terms of order $\alpha^{4} Z^{6}$ and $\alpha^{4} Z^{5}$. Any difference between the two calculations should therefore scale as $\alpha^{4} Z^{4}$, at least through the intermediate range of $Z$.

Persson et al. states that the missing correlation effects in their two-electron QED calculations is estimated to be of the order of $0.1 \mathrm{eV}$ for all elements. Formally this should only be applied to the range of elements $32<Z<92$. The associated uncertainties for $Z<32$ are unknown, but could be expected to increase in this regime. In the calculations of Drake, the uncertainty due to relativistic correlation effects in QED scales as $\alpha^{4} Z^{4}$. The sources of the uncertainty are quite different in the calculations of Drake and of Persson et al. The lowest-order Lamb shift is of order $\alpha^{3} Z^{4}$, and so the leading two-electron correction is of order $\alpha^{3} Z^{3}$, i.e., smaller by a factor of $1 / Z$. Higher-order correlation effects contribute further terms of order $\alpha^{3} Z^{2}, \alpha^{3} Z, \ldots$. In the calculations of Persson et al., it is the uncertainty in these correlation corrections to the lowest-order (in $\alpha$ ) Lamb shift that accounts for their estimate of $0.1 \mathrm{eV}$ for all elements. In contrast, the calculation of Drake accurately sums this entire series of terms, but there are relativistic correlation corrections to the QED shift of order $\alpha^{4} Z^{4}, \alpha^{5} Z^{5}, \ldots$, not included in this calculation. This accounts for Drake's error estimate of $1.2(Z / 10)^{4} \mathrm{~cm}^{-1}$ [5], scaling as $Z^{4}$. It has been suggested that Drake's error estimate should be multiplied by 8 for the ground state, to take into account a $1 / n^{3}$ scaling.
In summary, the calculation of Drake accurately treats the $1 / Z$ expansion of the nonrelativistic two-electron QED shift, but not the $\alpha Z$ expansion of relativistic and correlation corrections. The calculation of Persson et al. is improved for the $\alpha Z$ expansion, but not the $1 / Z$ expansion of the nonrelativistic correlation terms. For larger $Z$ the calculations of Persson et al. may be preferred, and for smaller $Z$ the calculations of Drake may be preferable.

\section{Excited-state QED}

Measuring the heliumlike $n=2$ to $n=1$ transitions can be sensitive to the excited-state QED contributions if the precision is sufficient. The QED contribution to the $1 s 2 s{ }^{3} S_{1}$ is $0.333 \mathrm{eV}$ from Drake [5] or $65 \mathrm{ppm}$ of the transition energy. Our measurement of the $z\left(1 s 2 s^{3} S_{1} \rightarrow 1 s^{2}\right)$ transition is sensitive to the $1 s 2 s^{3} S_{1}$ QED contribution at the $40 \%$ level. Most previous experiments in the medium $Z$ region have only measured the $w$ transition, while others have only measured the $w$ and close-lying $x$ and $y$ lines. Ours is one of the most precise measurements of the $z$ transition in medium $Z$ ions.

Of course, the result is a direct measure of the $1 s 2 s{ }^{3} S_{1}$ $\rightarrow 1 s^{2}$ QED contribution. Most such measurements are insensitive to the excited-state $(2 s$ or $2 p$ ) QED contribution. However, our measurement is indeed sensitive to the excited-state QED contribution at the $40 \%$ level. Our result is comparable to the 51-ppm measurement for $Z=32$ [13] which tests the $1 s 2 s{ }^{3} S_{1}$ QED contribution at the $48 \%$ level.

\section{Previous heliumlike vanadium observations}

Four other observations of heliumlike vanadium spectra have been reported (see Table IV). Two of these [38,12] were relative measurements to the $w$ line and, as such, cannot be compared to absolute measurements of the $w$ line. These relative observations include a study of heliumlike vanadium at an EBIT [12] and are the only prior observations of the heliumlike resonance lines other than the $w$ line (the $x, y$, and $z$ lines). 
Two measurements $[39,11]$ were conducted at a lowinductance vacuum spark plasma and a tokamak plasma, respectively. In both cases only the $w$ line was reported. The first study by Aglitsky et al. [39] in 1988 was part of a broad survey of resonance transitions in heliumlike ions ( $Z$ =16-39). A double Johann spectrograph was used to record spectra. Characteristic $K$ lines were used for calibration based upon reference wavelengths of Cauclois [40]. The precision reported for the $w$ transition in vanadium was 105 ppm.

The second study by Beiersdorfer and co-workers [11] was part of a survey of medium $Z$ elements in the heliumlike isoelectronic sequence in tokamak plasmas at the Princeton Large Torus (PLT). The uncertainty reported for vanadium was $40 \mathrm{ppm}$. Close-lying Lyman series lines were used for calibration. Shorter-wavelength calibration lines and heliumlike resonance lines were observed in second-order diffraction. As discussed in Sec. III, significant systematic shifts can occur in measurements that utilize several diffraction orders unless properly accounted for. The theoretical values of Mohr [41] were used in that analysis for the hydrogenic transitions and therefore QED theory was assumed to be correct [11]. A review of hydrogenic medium $Z$ measurements demonstrates that the experimental evidence is incomplete in this region of $Z$.

While several high precision absolute measurements of hydrogenic transitions have been reported for $\operatorname{argon}(Z$ $=18$, e.g., Marmar et al. [42] and Beyer et al. [43]) and iron $(Z=26$, Chantler [27]), the elements with $Z=19$ to $Z=25$ have not been measured precisely and absolutely and in some cases have not been measured at all. For example, the Lyman $\alpha$ transitions in vanadium have only been investigated recently [44]. The practice of using internal reference lines based upon QED theory, while having spectroscopic advantages, cannot reliably be used to infer conclusions about QED contributions in two-electron systems.

Dielectronic satellite lines are often associated with heliumlike transitions particularly in plasmas where a wide range of electron energies are present. Satellite contamination in hot plasmas is often the major limiting uncertainty on the precision of energy measurements [45]. Bulk motions within the plasma can also be very fast and vary between charge states [46]. This may be contrasted to the situation in an EBIT where very low satellite contamination can be achieved and Doppler shifts are negligible.

The measurements of the $w$ resonance line in heliumlike vanadium $[39,11]$ are shown in Table IV and plotted in Fig. 6 where they can be compared to current theory and other experiments including this work. The results of Aglitsky et al. [39] lie higher in energy than theory but within experimental error. The measurements of Beiersdorfer et al. [11] across the range of $Z$ observed were consistently higher than theoretical energies but most, including vanadium, were individually within experimental errors of theory. It was claimed that the comparison shows that there are systematic differences between data and predictions, which indicate a need to include additional corrections in the calculations [11]. The implication of this series of measurements was that there was a systematic offset between theory and experiment, and that the uncertainty in the offset was smaller by roughly a factor of $\sqrt{N}$ than that of the individual measurements [more precisely, the mean offset of the six measurements is $34(15) \mathrm{ppm}]$. Our result for the $w$ line in vanadium indicates there is no systematic offset from theory; our value for the offset, $-10(27) \mathrm{ppm}$, differs from that inferred from the measurements of Beiersdorfer et al. by 44(27) ppm.

\section{E. Other absolute medium $Z$ measurements}

A survey of experimental measurements across medium $Z$ heliumlike resonance lines is shown in Table III and Fig. 6 and compared with recent theory. The most precise absolute measurement of medium $Z$ heliumlike ions is attributed to Deslattes and co-workers [47] with a $12 \mathrm{ppm}$ measurement of the $w$ transition in argon. The $x$ and $y$ transitions were also measured in this work to similar accuracy, but the $z$ transition was not observed. Our methodology is similar to that of Deslattes et al. in the use of an external x-ray calibration standard lying close to the wavelength of interest. The recoilion experimental method used therein also eliminates the need for Doppler corrections and uncertainties in that work as opposed to other experiments but is comparable to our work on the EBIT. A novel experimental arrangement produced $\mathrm{Ar}^{16+}$ by collisions of $\mathrm{MeV} \mathrm{U}^{66+}$ ions with an argongas target [43]. A Johann curved crystal spectrometer was employed with a position-sensitive proportional counter of the backgammon type (both the spectrometer and detector are similar to those used in this research). This spectroscopy method may have systematic errors associated with the geometry and detector type and position as discussed above (Sec. III). Three-electron satellite contamination in these observations was considerably reduced by adjusting the gas target pressure. The contribution of the residual satellite contamination to the overall precision of wavelength determination was estimated to be $3 \mathrm{ppm}$. However, as with all spectra with strong satellites, the limiting uncertainty is difficult to quantify. Argon is at the lower end of the medium $Z$ elements where QED effects are smaller relative to the transition energies. This very precise measurement of argon [47] is within experimental uncertainty of the unified [5] and all orders (AO) [6] calculations, but excludes the configuration-interaction (CI) [7] and multiconfiguration Dirac-Fock (MCDF) [9] calculations, as shown in Table III.

\section{F. Intensities of $1 s 2 p^{3} P_{1} \rightarrow 1 s^{2}$ and $1 s 2 p{ }^{3} P_{0} \rightarrow 1 s^{2}$ transitions}

The probability of the $1 s 2 p^{3} P_{0} \rightarrow 1 s^{2}$ transition reveals information about the dominant processes in an electronbeam ion trap. In helium, the $1 s 2 p{ }^{3} P_{0}$ level can only decay to the ground state by two or three photon processes, $E 1 M 1$ or $3 E 1$, respectively, but the probability is very low for all $Z$ [48]. Vanadium has a finite nuclear moment $(7 / 2 \hbar$ [49]), so the transition from the $1 s 2 p{ }^{3} P_{0}$ state to the ground state $\left(1 s 2{ }^{1} S_{0}\right)$ can also occur via the hyperfine interaction [50]. Mohr has calculated the $1 s 2 p{ }^{3} P_{0} \rightarrow 1 s^{2}$ transition rate to be $10.5 \times 10^{9} \mathrm{~s}^{-1}$ in vanadium [50]. 
TABLE V. Experimental determinations of the relative intensity of the strong $y\left(1 s 2 p{ }^{3} P_{1} \rightarrow 1 s^{2}\right)$ component compared to the total intensity of the blend ( $y$ and $1 s 2 p^{3} P_{0} \rightarrow 1 s^{2}$ ), using theoretical energies of Drake, Ref. [5].

\begin{tabular}{lccc}
\hline \hline$y \%$ of total blend intensity & TFR tokamak [38] & LLNL EBIT [12] & NIST EBIT $^{\mathrm{a}}$ \\
\hline Normalized to experimental $w$ line & & $99 \% \pm 15 \%$ & $94 \% \pm 12 \%$ \\
Normalized to theoretical $w$ line & $44 \% \mathrm{~b}$ & $87 \% \pm 15 \%$ & $97 \% \pm 12 \%$ \\
\hline \hline
\end{tabular}

a NIST EBIT: This work.

${ }^{\mathrm{b}}$ Relative measurement, no errors were published.

For vanadium, earlier theoretical work has claimed a ratio of $4: 1$ for the intensity of the $y\left(1 s 2 p^{3} P_{1} \rightarrow 1 s^{2}\right)$ transition relative to the $1 s 2 p^{3} P_{0} \rightarrow 1 s^{2}$ transition based upon electron-impact excitation cross-section calculations [12]. The calculation of Mohr combined with the $y$ transition rate $\left(1.638 \times 10^{13} \mathrm{~s}^{-1}\right)$ of Drake [51], and assuming equal initialstate populations, results in a ratio of $1560: 1$. The competing $1 s 2 p{ }^{3} P_{0} \rightarrow 1 s 2 s^{3} S_{1}$ rate is only $2 \%$ of the $1 s 2 p{ }^{3} P_{0}$ $\rightarrow 1 s^{2}$ transition rate so it does not influence the result.

The hyperfine-induced decay of the ${ }^{3} P_{0}$ level in vanadium cannot be resolved from the $y\left({ }^{3} P_{1}\right)$ transition in any available experimental method $[12,38]$. The relative intensities of the ${ }^{3} P_{0}$ and $y$ transitions therefore shift the observed centroid. The energy separation between ${ }^{3} P_{0}$ and $y$ lines is $1.47 \mathrm{eV}$ in vanadium which is equivalent to $290 \mathrm{ppm}$ of the energy of these transitions.

We determine the relative intensity of the $y$ component compared to the blend to be $94 \pm 12 \%$ (and consequently the ${ }^{3} P_{0}$ component is $6 \pm 12 \%$ ) based on the theoretical energies of Drake [5] and the experimentally determined centroid position of the blend. The width of each component is also consistent: $\quad w=3.82(29) \mathrm{eV}, \quad x=4.3(9) \mathrm{eV}, \quad y-{ }^{3} P_{0}$ $=4.1(7) \mathrm{eV}$, and $z=3.27(38) \mathrm{eV}$. There is no direct evidence for individual high- $n$ or recombination satellite contamination. The width of the $y-{ }^{3} P_{0}$ blend is within $1 \sigma$ of that of the other resonances and narrower than the (weak) $x$ line. This is consistent with our findings for the ratio of intensities based upon the centroid position and suggests an almost complete absence of the ${ }^{3} P_{0}$ transition. The results for position and width of the $y-{ }^{3} P_{0}$ blend from a second study of vanadium at the NIST EBIT agree with those of the first study, although the statistical uncertainties are larger.

Our absolute experimental determination of the relative intensity of the $y$ component is compared to relative measurements $[12,38]$ of the heliumlike resonance lines in vanadium in Table V. Achard et al. [38] results are substantially different from recent theory (see $d E$ values in Table IV) and were conducted at a tokamak plasma where satellite contamination was intense.

A comparison between EBIT experiments and their respective measurements is more revealing. The results from the Livermore EBIT [12] for the relative intensity of the $y$ component compared to the total blend intensity are presented in Table V. We have presented the relative intensity, firstly, based upon the absolute experimental results compared with theory and secondly based upon relative differences from the $w$ line and compared with differences as calculated in theory (results are normalized to the theoretical position of the $w$ line). Relative intensities of the $y$ component derived from the EBIT measurements range from $87 \%$ to $99 \%$ (with $15 \%$ uncertainty) for the Livermore EBIT and from $94 \%$ to $97 \%$ for the NIST EBIT, depending upon normalization.

Drake [51] has suggested that differences between EBIT results for the ratio may be due to pressure differences because low-pressures and steady-state conditions could lead to a build up of the ${ }^{3} P_{0}$ population relative to the ${ }^{3} P_{1}$ state. The experimental evidence from both EBIT studies supports an almost complete absence of the ${ }^{3} P_{0}$ transition and the results from the NIST EBIT are within experimental uncertainty of Drake's prediction (1560:1) [51] but exclude the earlier theoretical calculation of $4: 1$ [12]. The differences between EBIT results are within experimental errors. We conclude that the differences in EBIT experimental conditions have had a negligible effect on wavelength determination. This supports the value of absolute measurements from an EBIT source for precision QED investigations.

\section{CONCLUSIONS}

The use of well-determined reference wavelengths has been crucial to this research, and has led to the first absolute determination of heliumlike resonance lines in vanadium using an EBIT. The uncertainties of the $w\left(1 s 2 p^{1} P_{1} \rightarrow 1 s^{2}\right)$ and $z\left(1 s 2 s^{3} S_{1} \rightarrow 1 s^{2}\right)$ lines are 27 and $28 \mathrm{ppm}$, respectively, and the measurements of all the transitions are a 5.7$8 \%$ test of QED contributions. The results are in accord with, but do not discriminate between, current theories of Drake [5] and Plante et al. [6] which differ by $0.01 \mathrm{eV}$. The measurement of the $z\left(1 s 2 s^{3} S_{1} \rightarrow 1 s^{2}\right)$ transition is sensitive to the $1 s 2 s{ }^{3} S_{1}$ QED contribution at the $40 \%$ level.

The absolute calibration is achieved by using a spread of characteristic wavelengths $(K \alpha$ and $K \beta)$ to map out and rigorously determine the dispersion function of the spectrometer. We have developed the dynamical diffraction theory necessary to evaluate systematic shifts at the precision level required to test QED. Systematic shifts associated with the shape and location of the detector used in the Johann geometry have been evaluated and reduced to a level that is comparable to the uncertainty of the reference wavelengths used for calibration.

Our measurement of the $w$ line in vanadium is not in accord with previous measurements of medium- $Z$ heliumlike ions (Beiersdorfer et al. [11]) conducted at a tokamak plasma 
and the EBIT at LLNL [13] which reported a trend of experimental energies above theory. The location and width of the $y-{ }^{3} P_{0}$ blend implies a relative intensity of the $y$ component of $94 \%$ compared to the total blend intensity. This is in accord with recent predictions based upon transition rates but in disagreement with an earlier claim.

The unique spectroscopic advantages of the EBIT have been crucial in the success of these QED investigations, allowing Doppler-free and low-satellite contamination spectra to be measured. The benefits of absolute calibration com- bined with rigorous diffraction theory in precision tests of QED have been demonstrated.

\section{ACKNOWLEDGMENTS}

We would like to acknowledge the assistance of A. Henins, R. D. Deslattes, and L. Ratliff during the experiments at NIST. We thank G. W. Drake for helpful discussions and comments. This research was supported by the Australian Research Council.
[1] G. W. Series, The Spectrum of Atomic Hydrogen: Advances (Utopia, Singapore, 1988).

[2] P. J. Mohr, in Atomic, Molecular, \& Optical Physics Handbook (AIP, Woodbury, NY, 1996), Chap. 28.

[3] R. Marrus Physics of Highly-Ionized Atoms (Plenum, New York, 1989).

[4] G. W. F. Drake, Phys. Rev. A 19, 1387 (1979).

[5] G. W. Drake, Can. J. Phys. 66, 586 (1988).

[6] D. R. Plante, W. R. Johnson, and J. Sapirstein, Phys. Rev. A 49, 3519 (1994).

[7] K. T. Cheng, M. H. Chen, W. R. Johnson, and J. Sapirstein, Phys. Rev. A 50, 247 (1994).

[8] M. H. Chen, K. T. Cheng, and W. R. Johnson, Phys. Rev. A 47, 3692 (1993).

[9] P. Indelicato, F. Parente, and R. Marrus, Phys. Rev. A 40, 3505 (1989); P. Indelicato, Nucl. Instrum. Methods Phys. Res. B 31, 14 (1988); P. Indelicato, O. Gorceix, and J. P. Desclaux, J. Phys. B 20, 651 (1987).

[10] H. Persson, S. Salomonson, P. Sunnergren, and I. Lindgren, Phys. Rev. Lett. 76, 204 (1996).

[11] P. Beiersdorfer, M. Bitter, S. von Goeler, and K. W. Hill, Phys. Rev. A 40, 150 (1989).

[12] P. Beiersdorfer, M. H. Chen, R. E. Marrs, M. B. Schneider, and R. S. Walling, Phys. Rev. A 44, 396 (1991).

[13] S. MacLaren, P. Beiersdorfer, D. A. Vogel, D. Knapp, R. E. Marrs, K. Wong, and R. Zasadzinki, Phys. Rev. A 45, 329 (1992).

[14] A. H. Gabriel, Mon. Not. R. Astron. Soc. 160, 99 (1972).

[15] E. Takács, E. S. Meyer, J. D. Gillaspy, J. R. Roberts, C. T. Chantler, L. T. Hudson, R. D. Deslattes, C. M. Brown, J. M. Laming, J. Dubau, and M. K. Inal, Phys. Rev. A 54, 1342 (1996).

[16] J. D. Gillaspy, et al., Phys. Scr. T59, 392 (1995).

[17] J. D. Gillaspy, Phys. Scr. T65, 168 (1996).

[18] A. Henins, Rev. Sci. Instrum. 58, 1173 (1987).

[19] B. P. Duval, J. Barth, R. D. Deslattes, A. Henins, and G. G. Luther, Nucl. Instrum. Methods Phys. Res. A 222, 274 (1984).

[20] D. J. Paterson, C. T. Chantler, C. Tran, L. T. Hudson, F. G. Serpa, and R. D. Deslattes, Phys. Scr. T73, 400 (1997).

[21] C. T. Chantler, D. J. Paterson, L. T. Hudson, F. G. Serpa, J. D. Gillaspy, and E. Takács, Phys. Scr. T80, 440 (1999).

[22] J. A. Bearden, Rev. Mod. Phys. 39, 78 (1967).

[23] C. T. Chantler and R. D. Deslattes, Rev. Sci. Instrum. 66, 5123 (1995).
[24] J. P. Briand, M. Tavernier, R. Marrus, and J. P. Desclaux, Phys. Rev. A 29, 3143 (1984).

[25] H. F. Beyer and D. Liesen, Nucl. Instrum. Methods Phys. Res. A 272, 895 (1988).

[26] P. Beiersdorfer, R. E. Marrs, J. R. Henderson, D. A. Knapp, M. A. Levine, D. B. Platt, M. B. Schneider, D. A. Vogel, and K. L. Wong, Rev. Sci. Instrum. 61, 2338 (1990).

[27] C. T. Chantler, D. Phil. thesis, Oxford University, 1990 (unpublished).

[28] C. T. Chantler, J. Appl. Crystallogr. 25, 674 (1992); 25, 698 (1992).

[29] M. Deutsch and M. Hart, Phys. Rev. B 26, 5558 (1982).

[30] J. Härtwig, G. Hölzer, J. Wolf, and E. Förster, J. Appl. Crystallogr. 26, 539 (1993).

[31] J. Schweppe, R. D. Deslattes, T. Mooney, and C. J. Powell, J. Electron Spectrosc. Relat. Phenom. 67, 463 (1994).

[32] R. D. Deslattes, E. G. Kessler, P. Indelicato, and E. Lindroth, International Tables for Crystallography (Kluwer Academic Press, Stockholm, 1999), Vol. C, Sec. 4.2.2.

[33] R. D. Deslattes, Phys. Scr. T46, (1993).

[34] R. D. Deslattes, E. G. Kessler, W. C. Sauder, and A. Henins, Ann. Phys. (N.Y.) 129, 378 (1980).

[35] G. Hölzer, M. Fritsch, M. Deutsch, J. Härtwig, and E. Förster, Phys. Rev. A 56, 4554 (1997).

[36] R. E. Marrs, S. R. Elliot, and T. Stöhlker, Phys. Rev. A 52, 3577 (1995).

[37] W. R. Johnson and G. Soff, At. Data Nucl. Data Tables 33, 405 (1985); P. J. Mohr, Phys. Rev. A 46, 4421 (1992).

[38] M. H. Achard et al., M. Cornille, J. Dubau, and M. Loulergue TFR Group, Phys. Rev. A 32, 3000 (1985).

[39] E. V. Aglitsky, P. S. Antsiferov, S. L. Mandelstam, A. M. Panin, U. I. Safronova, S. A. Ulitin, and L. A. Vainshtein, Phys. Scr. 38, 136 (1988).

[40] Y. Cauclois and C. Senemaud, Wavelength of X-ray Emission Lines and Absorption Edges (Pergamon, London, 1988).

[41] P. J. Mohr, At. Data Nucl. Data Tables 29, 454 (1983).

[42] E. S. Marmar, J. E. Rice, E. Källne, J. Källne, and R. E. LaVilla, Phys. Rev. A 33, 774 (1986).

[43] H. F. Beyer, R. D. Deslattes, F. Folkmann, and R. E. La Villa, J. Phys. B 18, 207 (1985).

[44] D. J. Paterson, Ph.D. thesis, University of Melbourne, 1999 (unpublished).

[45] E. Träbert, The Spectrum of Atomic Hydrogen: Advances (Utopia, Singapore, 1988), Chap. 6. 
[46] E. Källne, J. Källne, P. Richard, and M. Stöckli, J. Phys. B 17, L115 (1984).

[47] R. D. Deslattes, H. F. Beyer, and F. Folkmann, J. Phys. B 17, L687 (1984).

[48] H. F. Beyer, H. J. Kluge, and V. P. Shevelko, X-Ray Radiation of Highly Charged Ions (Springer, Berlin, 1997).
[49] R. C. Weast, Handbook of Physics and Chemistry (CRC Press, Boca Raton, Fl, 1985).

[50] P. J. Mohr, in Beam-Foil Spectroscopy, edited by I. A. Sellin and D. J. Pegg (Plenum, New York, 1976).

[51] G. W. Drake (personal communication). 